\title{
Hypothermic Neuronal Rescue from Infection-Sensitised Hypoxic-Ischaemic Brain Injury Is Pathogen Dependent
}

\author{
Mari Falck $^{\mathrm{a}}$ Damjan Osredkar ${ }^{\mathrm{b}}$ Elke Maes $^{\mathrm{a}}$ Torun Flateb $\varnothing^{\mathrm{a}}$ \\ Thomas Ragnar Wood ${ }^{a}$ Hemmen Sabir ${ }^{a, c}$ Marianne Thoresen ${ }^{a, d}$

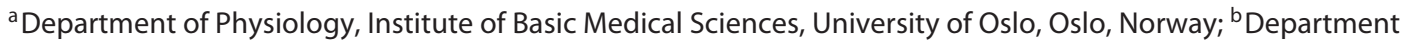 \\ of Paediatric Neurology, University Children's Hospital, Ljubljana, Slovenia; 'Department of General Pediatrics, \\ Neonatology and Pediatric Cardiology, University Children's Hospital, Heinrich Heine University, Düsseldorf, \\ Germany; ${ }^{d}$ Neonatal Neuroscience, School of Clinical Sciences, University of Bristol, Bristol, UK
}

\section{Keywords}

Perinatal brain injury $\cdot$ Asphyxia $\cdot$ Hypothermia therapy . Neuroprotection · Infection · Inflammation · Gram-positive pathogens $\cdot \mathrm{PAM}_{3} \mathrm{CSK}_{4}$. Lipopolysaccharide

\begin{abstract}
Perinatal infection increases the vulnerability of the neonatal brain to hypoxic-ischaemic (HI) injury. Hypothermia treatment $(\mathrm{HT})$ does not provide neuroprotection after pre-insult inflammatory sensitisation by lipopolysaccharide (LPS), a gram-negative bacterial wall constituent. However, earlyonset sepsis in term babies is caused by gram-positive species in more than $90 \%$ of cases, and neuro-inflammatory responses triggered through the gram-negative route (Tolllike receptor 4, TLR-4) are different from those induced through the gram-positive route via TLR-2. Whether grampositive septicaemia sensitises the neonatal brain to hypoxia and inhibits the neuroprotective effect of $\mathrm{HT}$ is unknown. Seven-day-old Wistar rats $(n=178)$ were subjected to intraperitoneal injections of $\mathrm{PAM}_{3} \mathrm{CSK}_{4}(1 \mathrm{mg} / \mathrm{kg}$, a synthetic TLR2 agonist) or vehicle $(0.9 \% \mathrm{NaCl})$. After an 8 -h delay, the left carotid artery was ligated followed by 50 min of hypoxia ( $8 \%$ $\mathrm{O}_{2}$ ) at a rectal temperature of $36^{\circ} \mathrm{C}$. Pups received a 5 -h treat-
\end{abstract}

๑ 2017 S. Karger AG, Basel

\section{KARGER}

E-Mail karger@karger.com

www.karger.com/dne ment of normothermia $\left(\mathrm{NT}, 37^{\circ} \mathrm{C}\right)$ or $\mathrm{HT}\left(32^{\circ} \mathrm{C}\right)$ immediately after the insult. Brains were harvested after 7 days' survival for hemispheric and hippocampal area loss analyses and immunolabelling of microglia (Iba1) and hippocampal neurons (NeuN). Normothermic PAM $\mathrm{CSK}_{4}$-injected animals showed significantly more brain injury than vehicle animals $(p=$ 0.014). Compared to NT, HT significantly reduced injury in the $\mathrm{PAM}_{3} \mathrm{CSK}_{4}$-injected animals, with reduced area loss $(p<$ $0.001)$, reduced microglial activation $(p=0.006)$, and increased neuronal rescue in the CA1 region $(p<0.001)$. Experimental induction of a sepsis-like condition through the gram-positive pathway sensitises the brain to $\mathrm{HI}$ injury. HT was highly neuroprotective after the $\mathrm{PAM}_{3} \mathrm{CSK}_{4}$-triggered injury, suggesting HT may be neuroprotective in the presence of a gram-positive infection. These results are in strong contrast to LPS studies where HT is not neuroprotective.

(C) 2017 S. Karger AG, Basel

\section{Introduction}

Perinatal hypoxic-ischaemic (HI) brain injury remains one of the major causes of long-term neurological disability or death in term newborns [1]. Perinatal infection is a
Marianne Thoresen, $\mathrm{MD}, \mathrm{PhD}$

Department of Physiology, Institute of Basic Medical Sciences

University of Oslo, Domus Medica, Boks 1072 Blindern NO-0316 Oslo (Norway)

E-Mail marianne.thoresen@ medisin.uio.no 
risk factor for cerebral palsy and long-term disability [24], and systemic inflammation also lowers the threshold at which an $\mathrm{HI}$ insult leads to permanent neuronal injury [5-7]. Several small and large animal studies have demonstrated the infection-induced vulnerability of the brain to hypoxia, and investigated the mechanisms behind [811]. Interestingly, a generalised systemic inflammatory activation seems to be sufficient to cause this sensitisation, even in the absence of the pathogen itself. Chau et al. [12] showed that meningitis is not a prerequisite to increase the susceptibility of the brain to HI injury, and most clinical studies linking severity of brain injury to perinatal infection have instead examined pro-inflammatory cytokines or clinical signs of perinatal infection, such as maternal pyrexia or clinical chorio-amnionitis $[3,13]$. Furthermore, the success rate of pathogen isolation from the blood of neonates with clinical infection is poor at only 6\% [14]. Preclinical animal models of simulated infection in the setting of HI injury often use inflammatory triggers like lipopolysaccharide (LPS), a constituent of gram-negative bacterial membranes, in place of the complete bacteria $[8,9,15,16]$. Systemic activation of immune cells will not only induce an inflammatory cascade in peripheral blood, but also induces inflammatory activation in brain tissue. The elevated cytokines and activated microglia elicit what is referred to as the infectionsensitised immature brain [7, 16-18].

For term neonates with moderate and severe HI encephalopathy as a result of HI brain injury, hypothermia treatment (HT) is standard of care, and our only current treatment option [19]. With a number needed to treat of $8,45-50 \%$ of encephalopathic term babies will still die or have long-term disability despite active HT therapy [20]. Based on a diverse range of clinical and preclinical studies, doubt exists as to whether HT is neuroprotective in infants with HI encephalopathy where perinatal infection is a comorbidity $[6,21,22]$. We have recently shown experimentally that HT is not neuroprotective after preinsult inflammatory sensitisation with LPS in a postnatal day 7 (P7) rat model of unilateral HI brain injury [8]. LPS triggers the immune system primarily by binding to Tolllike receptor (TLR) 4 but is likely to only represent infections caused by gram-negative bacteria which contain LPS in their cell wall [23]. However, in term neonates in developed countries where HT is standard of care, culture-positive sepsis has been shown to be caused by gram-positive bacterial species in $>90 \%$ of cases [14]. Peptidoglycans and lipoteichoic acid on the wall of grampositive bacteria trigger human immune responses by binding to TLR-2, and thereby induce a different path-

Pathogen-Dependent Hypothermic

Neuroprotection way to inflammatory activation [24-26]. We previously investigated the neuro-inflammatory responses in neonatal rat pups receiving systemic LPS, compared to those receiving the synthetic TLR-2 agonist $\mathrm{PAM}_{3} \mathrm{CSK}_{4}$ (PAM). Profound differences in temporal core temperature development, as well as in brain cytokine expression and inflammatory and apoptotic signal molecules were found, in response to the 2 different types of systemic inflammation [27].

Whether gram-positive septicaemia sensitises the neonatal brain to $\mathrm{HI}$ injury the way gram-negative septicaemia does, and whether it abolishes the neuroprotective effect of HT (as seen in LPS sensitisation), is not known.

We therefore investigated the sensitising effect of systemic TLR-2 activation on the neonatal rat brain, as a model of gram-positive systemic inflammation in the setting of HI brain injury, in the P7 rat. Additionally we investigated whether $\mathrm{HT}$ is neuroprotective in this doublehit setting.

\section{Materials and Methods}

Animals and Injections

All experiments were approved by the University of Oslo's Animal Ethics Research Committee and performed by individuals holding an approved license according to the Animal (Scientific Procedures) Act of 1986. Experiments were performed on P7 Wistar rats (Charles River Laboratories, Sulzfeld, Germany) of both sexes. All pups were kept in an animal facility with a 12-h dark:12-h light cycle at $19-21^{\circ} \mathrm{C}$ environmental temperature with food and water ad libitum.

To trigger inflammation through the TLR-2 pathway, we used a synthetically manufactured TLR-2 agonist - N-palmitoyl-S-(2,3bis(palmitoyloxy)-(2R,S-propyl)-R-cysteinyl-seryl-(lysyl)-3-lysine, $\mathrm{PAM}_{3} \mathrm{CSK}_{4}$ (PAM) (Vaccigrade, Sigma-Aldrich) - at a dose of $1 \mathrm{mg} / \mathrm{kg}$ body weight. PAM was initially dissolved in sterile LPSfree water and then diluted in sterile physiological saline $(0.9 \%$ $\mathrm{NaCl}$ ). The dose of PAM was based on previous publications on this agonist used in neonatal rodents [28-30], in combination with our own dose-response experiments (data not shown). Control groups received a single dose of sterile saline vehicle. All injections were given intraperitoneally in a volume of $10 \mu \mathrm{L} / \mathrm{g}$ body weight.

Animals were randomised across litter, sex, and weights before the experiments commenced, to one of the following treatment groups: vehicle injection (Veh) and normothermia (NT) (VehNT), PAM injection and NT (PAM-NT), Veh injection and HT (Veh-HT), and PAM injection and HT (PAM-HT).

\section{Surgical Procedures}

All experiments were performed as previously described for the LPS-sensitised modification of the Vannucci model of unilateral HI [8]. Briefly, at the start of each experiment, animals were injected with PAM or Veh according to randomisation. After an 8-h delay with their dams, pups were exposed to a mild HI insult (ligation of the left carotid artery under isoflurane anaesthesia followed
Dev Neurosci 2017;39:238-247

DOI: $10.1159 / 000455838$ 
by exposure to $8 \% \mathrm{O}_{2}$ for $50 \mathrm{~min}$ ). Immediately thereafter, pups received either of the 2 allocated treatments: $5 \mathrm{~h}$ of NT ( $\mathrm{T}_{\text {rectal }}$ $\left.37.0^{\circ} \mathrm{C}\right)$ or $\mathrm{HT}\left(\mathrm{T}_{\text {rectal }} 32.0^{\circ} \mathrm{C}\right)$.

During treatment, the core and surface temperatures of 2 "sentinel" pups from the Veh groups were continuously recorded in each chamber. Rectal temperature was maintained within $\pm 0.2^{\circ} \mathrm{C}$ of the target value using a continuous temperature recording (IT21; Physitemp Instruments, Clifton, NJ, USA), which servo-controlled a water-filled mat (CritiCool, MTRE, Yavne, Israel) on the floor of the chamber.

After the 5-h treatment period, pups were returned to their dams. Pups were sacrificed on postnatal day (P) 14 for further analyses.

\section{Histopathology and Area Loss Analyses}

At P14, animals were sacrificed by transcardiac perfusion-fixation with $10 \%$ neutral-buffered formalin under isoflurane $\mathrm{N}_{2} \mathrm{O}$ anaesthesia. Brains were harvested and kept in 10\% neutral-buffered formalin until further processing. Three-millimetre coronal blocks were cut using a standard rat matrix (ASI Instruments Inc., Warren, MI, USA) and embedded in paraffin. Five-micrometre slices were cut from the 2 neighbouring blocks best representing the cortex, hippocampus, basal ganglia and thalamus. These were stained with haematoxylin and eosin (HE) and scanned (Epson Perfection V750 Pro). Virtual slides were exported with 600 dpi resolution. Optical density and hemispheric area was analysed using Image J computer software (ImageJ, version 1.46r, National Institutes of Health, Bethesda, MD, USA). The ligated side was compared to the non-ligated side, and area loss of the ligated side was calculated by the formula $(1-$ [area left/area right] $) \times 100$. Percent hemispheric area loss at this level has previously been shown to be highly correlated with a formal neuropathology score and global degree of injury in this model [31].

Evaluation of hippocampal area loss was performed in the same way and calculated as: (1 - [area of left hippocampus/area of right hippocampus] $\times 100)$. A subset of the HE-stained sections was examined for hemispheric and hippocampal areas by 2 blinded assessors to check for interrater reliability.

\section{Immunohistochemistry}

For immunohistochemistry analysis, slides were prepared from paraffin-embedded sections as for HE staining. Antigen retrieval was then performed in citrate buffer solution at $\mathrm{pH}$ 6.0, using a PT link instrument (Dako, Glostrup, Denmark). After blocking with $10 \%$ goat serum, primary rabbit antibody against ionised calcium-binding adapter molecule 1 (Iba1; 1:1,000; WAKO), or mouse antibody against NeuN (1:500; Millipore), was applied overnight at $4^{\circ} \mathrm{C}$. In control brain sections, the primary antibodies were omitted. After rinsing with PBS, the slices were incubated for $1 \mathrm{~h}$ at room temperature with secondary Alexa Fluor 568 and/or 488 (Invitrogen, 1:500) antibodies. Finally, the slides were rinsed and coverslipped with ProLong Gold with DAPI (Invitrogen). Sections were scanned with a virtual microscopy scanner (Axio Scan Z1; Carl Zeiss, Jena, Germany) using the fluorescence mode with a plan apochromatic $\times 20$ lens. Virtual slides were exported as highresolution tiff images for further analysis.

To evaluate the effect of different treatments on neuronal loss, NeuN- and DAPI-positive cells in the CA1 region of the hippocampus were counted, as this region is known to be particularly vulnerable to hypoxia at P7 $[32,33]$. Aiming for a representative subset from each treatment group, the 10 animals closest to the median hemispheric area loss were selected for formal hippocampal neuron counting. Three non-overlapping fields of the CA1 region in the left hippocampi were assessed. Counting was performed by 2 individual observers blinded to the treatment group, and an average of the 2 was taken. The total number of neurons across the 3 fields of each hippocampus was summed and compared across groups.

To investigate inflammatory activation, staining for Iba1 (a microglial specific biomarker) was performed. Iba1-positive cells were separated from background and analysed by ImageJ. The summed colour intensity detected was calculated as an L/R hemispheric ratio and normalised to cross-sectional area before comparison across groups. The results from 2 blinded assessors were compared by calculating their correlation coefficient to validate the method.

\section{Data Analysis}

Statistical analyses were performed using GraphPad Prism version 6 (GraphPad Software Inc., La Jolla, CA, USA) and SPSS software version 22 (SPSS Inc., Chicago, IL, USA). As data were not normally distributed, the Kruskal-Wallis test was used for 4-group treatment comparison, and the Mann-Whitney U test was used for 2 -group comparisons to get exact 2 -tailed $p$ values. Graphical data are presented as median with $95 \%$ confidence intervals (CI). A $p$ value of $<0.05$ was considered as statistically significant.

\section{Results}

\section{Mortality and Exclusions}

Of the 218 pups initially included, no mortality was seen from injections alone. Four pups died during experimental surgical procedures and hypoxia. Pups carrying temperature probes in each experiment were excluded from further analysis $(n=16)$, because the stress of carrying the probe could influence the outcome [34]. A total of 198 pups were therefore included in the analysis. Twenty pups served as juvenile controls, without receiving an injection.

\section{Hemispheric Area Loss after Systemic Injections without HI}

To make sure a systemic inflammation triggered by injection of PAM does not create brain tissue loss on its own, P7 rat pups received injections according to the above-mentioned protocol with Veh $(n=22)$ or PAM $(n=23)$. Juvenile control animals of equivalent age $(n=$ 20) were used as controls. Total cross-sectional area was compared across groups, as well as right hemispheric area and left hemispheric area separately. There was no statistical difference between treatment groups in any of the analyses (Kruskal-Wallis test; $p=0.4,0.5$, and 0.3 , respectively) (data not shown). 


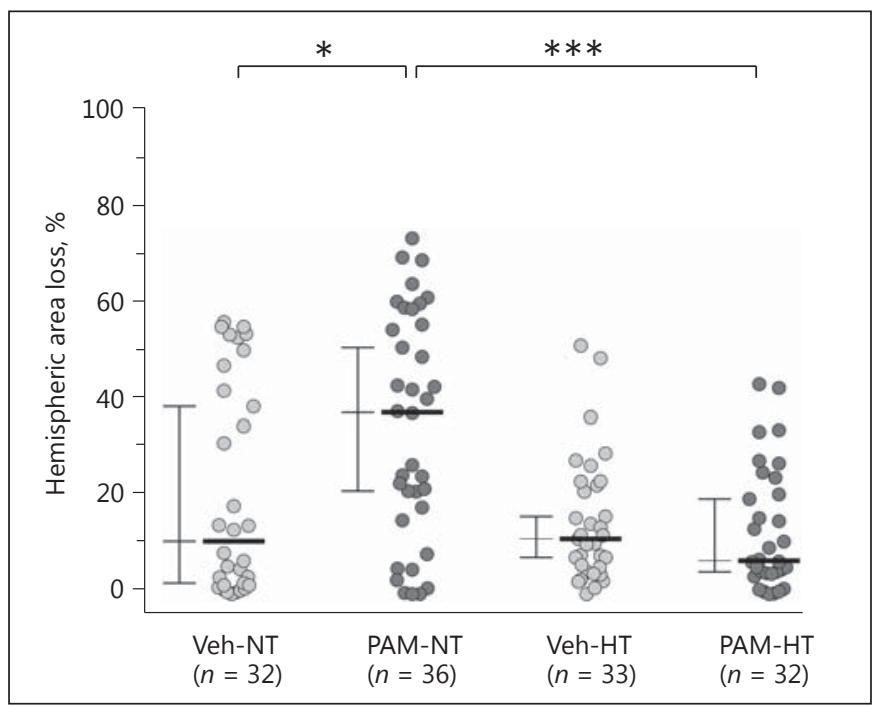

Fig. 1. Hemispheric area loss (\%). Bars show median with 95\% CI. P7 rat pups were injected intraperitoneally with vehicle (Veh) or $\mathrm{PAM}_{3} \mathrm{CSK}_{4}$ (PAM). After an 8-h delay, all pups had their left carotid artery ligated before $50 \mathrm{~min}$ of $8 \%$ hypoxia. Pups were randomised to $5 \mathrm{~h}$ of $\mathrm{NT}\left(37^{\circ} \mathrm{C}\right)$ or $\mathrm{HT}\left(32^{\circ} \mathrm{C}\right)$, with 7 days' survival. PAM-injected animals treated with NT (PAM-NT) had significantly more injury compared to the Veh-NT group. HT provided significant neuroprotection in the PAM-injected group (PAMHT). ${ }^{*} p=0.01,{ }^{* * *} p=0.0002$.

\section{Hypothermic Neuroprotection in PAM-Sensitised HI Injury}

In the NT-treated groups, animals receiving a single intraperitoneal injection of PAM prior to the $\mathrm{HI}$ insult were more vulnerable to $50 \mathrm{~min}$ of hypoxia and had significantly increased hemispheric area loss $(35.8 \%$, CI 20.4-48.6) compared to Veh-injected pups $(10.4 \%$, CI $2.1-37 ; p=0.01)$. Treating PAM-injected pups with HT reduced median area loss (6.6\%, CI 4.4-18.8) and thereby showed a significant neuroprotective effect compared to PAM animals treated with NT $(p=0.0002)$ (Fig. 1).

Also hippocampal area loss was significantly higher in PAM-NT pups (55.5\%, CI 26.3-69.2) compared to the Veh-NT group $(13.4 \%$, CI 3.1-40.2; $p=0.03)$. Significant hypothermic neuroprotection in the hippocampal region was seen in PAM-HT animals (8.2\%, CI 3.6-18.2; $p=0.003$ ) (Fig. 2). In the cortical area loss analyses we found the same differences, with PAM-NT pups having significantly more cortical tissue loss $(50.4 \%$, CI $25.1-$ 67.6) compared to the Veh-NT group (18.5\%, CI 2.4$54.2 ; p=0.03)$. Significant neuroprotection in the cortical region was seen in PAM-HT animals (8.2\%, CI 3.6-18.2; $p=0.003)$. Thalamic area loss was not significantly increased in the PAM-sensitised pups $(28.8 \%$, CI $13.2-$

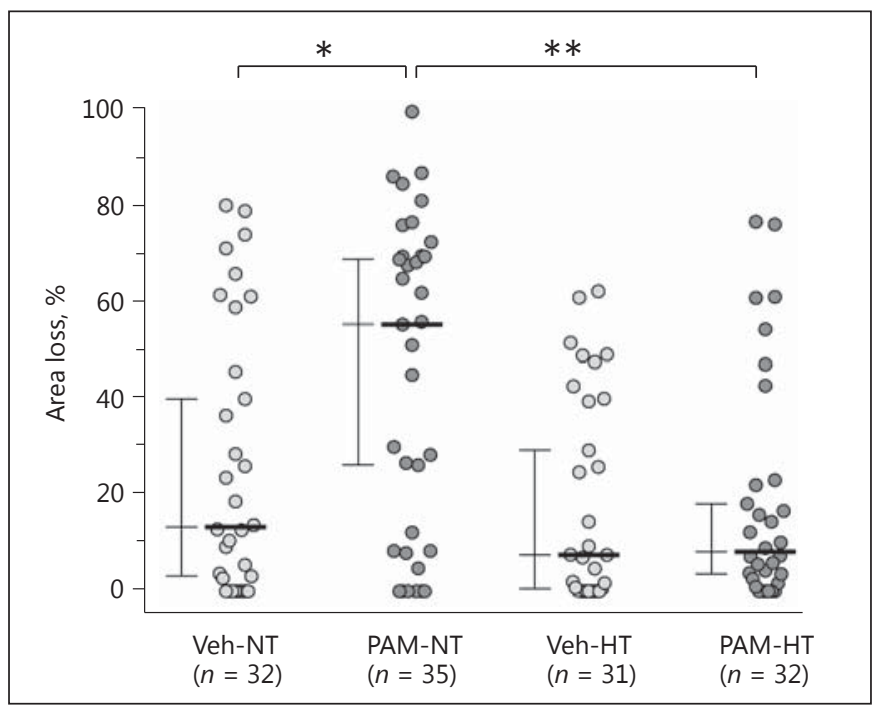

Fig. 2. Hippocampal area loss (\%). Bars show median with 95\% CI. $\mathrm{PAM}_{3} \mathrm{CSK}_{4}$-injected (PAM) pups receiving NT (PAM-NT) lost significantly more hippocampal tissue than vehicle-injected pups (Veh-NT) receiving the same treatment. HT was significantly neuroprotective in PAM-injected pups (PAM-HT). ${ }^{*} p=0.03,{ }^{* *} p=$ 0.003 .

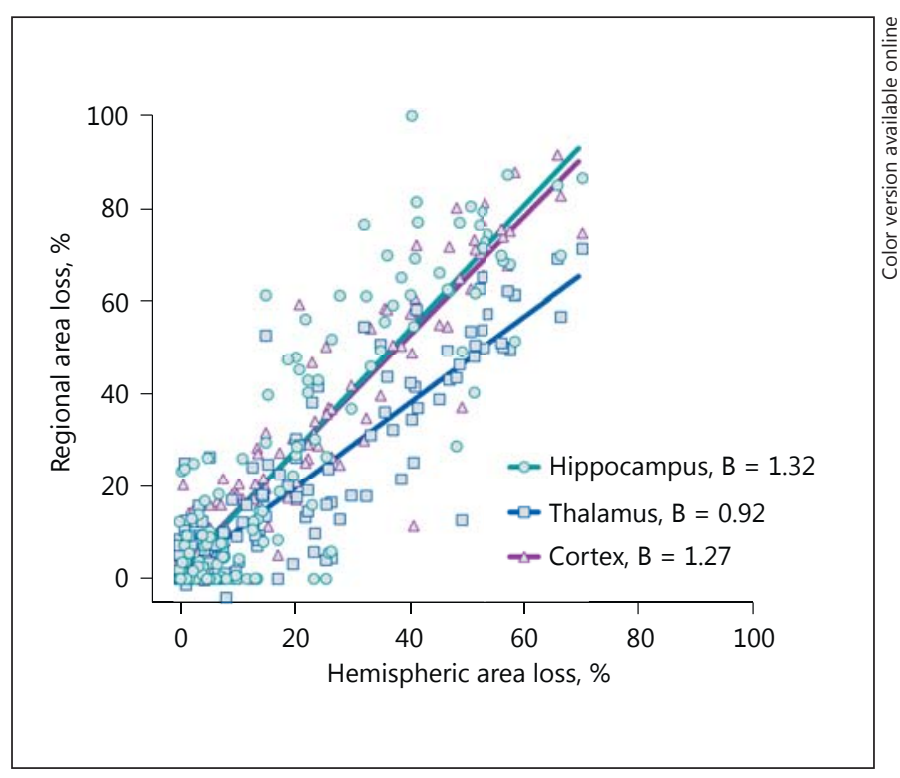

Fig. 3. Correlation between regional and hemispheric area loss. Symbols represent unique animals, with lines denoting the correlation between hemispheric area loss and area loss in the hippocampus (circles; $R^{2}=0.77$ ), thalamus (squares; $R^{2}=0.78$ ), or cortex (triangles; $R^{2}=0.89$ ).
Pathogen-Dependent Hypothermic Neuroprotection
Dev Neurosci 2017;39:238-247 DOI: $10.1159 / 000455838$ 

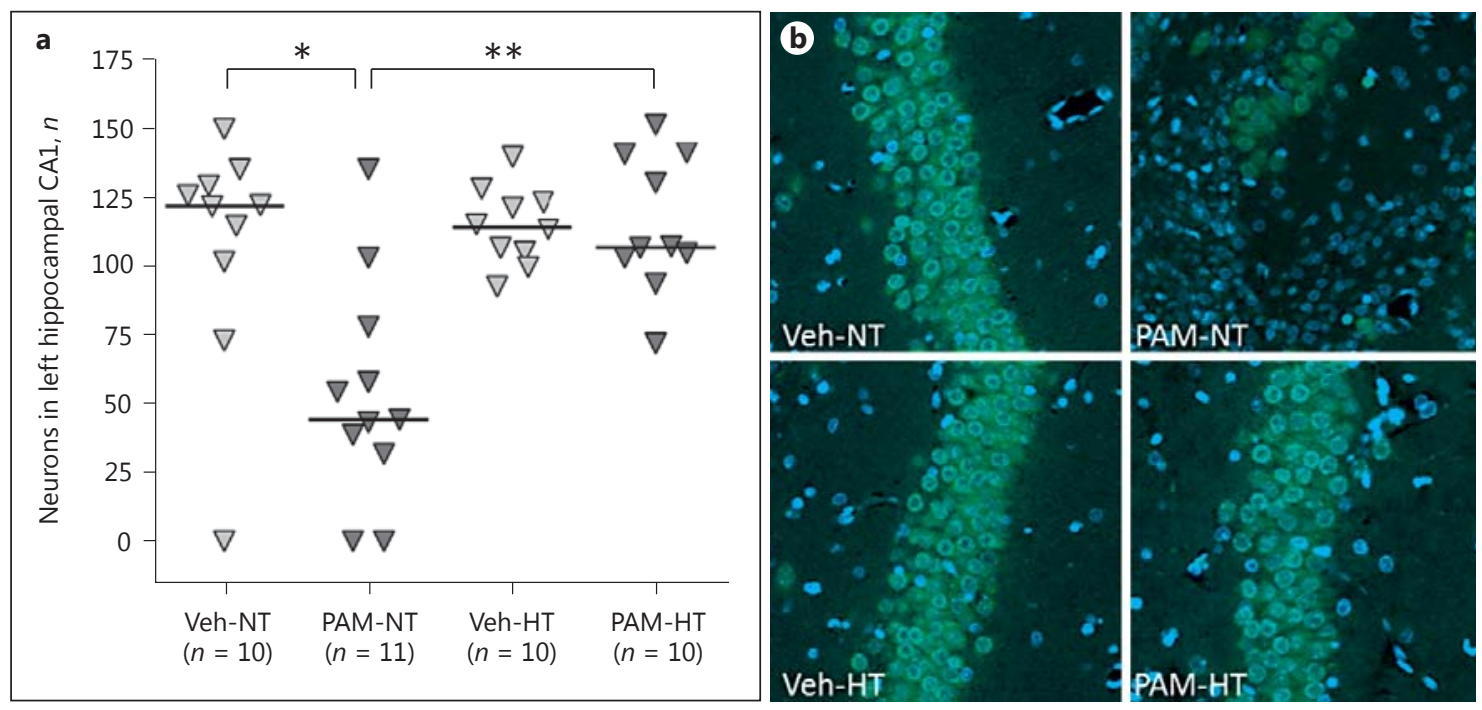

Fig. 4. Hippocampal neuron count. a Symbols represent the number of hippocampal neurons in the hippocampal CA1 region of each animal. Lines show the median. The neuron count was significantly lower in $\mathrm{PAM}_{3} \mathrm{CSK}_{4}$ injected (PAM) animals compared to vehicle-injected (Veh) animals in the NT groups. HT provides significant neuronal rescue in the PAM-sensitised animals. ${ }^{*} p=0.01$, ${ }^{* *} p=0.008$. b Representative images from the hippocampal CA1 region are shown from each experimental group.

46.3) compared to Veh-NT animals (14.2\%, CI 7-32.1). There was, however, a significant neuroprotective effect on thalamic tissue in the PAM-HT group (9.3\%, CI 3.2$18 ; p=0.01$ ).

There was no statistical difference between Veh-injected pups treated with NT and Veh animals receiving HT, neither with respect to hemispheric (10.4\%, CI 2.137 , vs. $10.9 \%$, CI 7.2-15.4) area loss nor to hippocampal (13.4\%, CI 3.1-40.2, vs. $7.6 \%$, CI $0.5-29.4)$, cortical (18.5\%, CI 2.4-54.2, vs. $15.9 \%$, CI 9.8-20.5), or thalamic (14.2\%, CI 7-32.1, vs. 9.3\%, CI 4.5-12.6) area loss.

Linear regression analysis showed a highly significant correlation between area loss in all 3 regions and hemispheric area loss (hippocampus: $R^{2}=0.77, p<0.0001$; cortex: $R^{2}=0.89, p<0.0001$; thalamus: $R^{2}=0.78, p<$ 0.0001 ), with hippocampal and cortical loss tending to be greater ( $\mathrm{B}=1.32$ and $\mathrm{B}=1.27$, respectively) than hemispheric area loss, while thalamic loss is slightly lower $(\mathrm{B}=0.92)$ (Fig. 3). This is in accordance with regional analysis of vulnerability in the Vannucci rat model when exposed to HI insult only [32].

\section{Hypothermia Provides Neuronal Rescue in the CA1}

Hippocampal Region

The total number of neurons in the CA1 region of the left hippocampus was counted in a subset of animals from all 4 treatment groups ( $n=10-11$ per group) (Fig. 4$)$. The number of neurons after a short $\mathrm{HI}$ insult was significantly lower in PAM-NT animals (44, CI 0-103) compared to Veh animals (122, CI 73-135; $p=0.01)$. Significant neuronal rescue was seen in the PAM-HT group (107, CI 94$141 ; p=0.0008)$. There was no difference between the 2 Veh-injected groups (NT: 121.8, CI 73-135, vs. HT: 114, CI 99.5-128).

\section{PAM-Induced Microglial Activation}

Iba1 upregulation was more pronounced and greater relative to the amount of remaining tissue, in PAM-NT animals $(p=0.035)$. Microglial activation was reversed in the PAM-HT group, with significantly reduced Ibal immunolabelling ( $p=0.006$ ) (Fig. 5). Microglial activation, with bigger somas and retracted dendritic processes, was morphologically visible around the left hemispheric lesions of all animals (Fig. 6).

\section{Discussion}

This study investigated the sensitising effect of systemic TLR-2 activation on the immature rat brain, combined with a mild unilateral $\mathrm{HI}$ insult. The motivation was to improve our preclinical model of infection-sensitised HI 


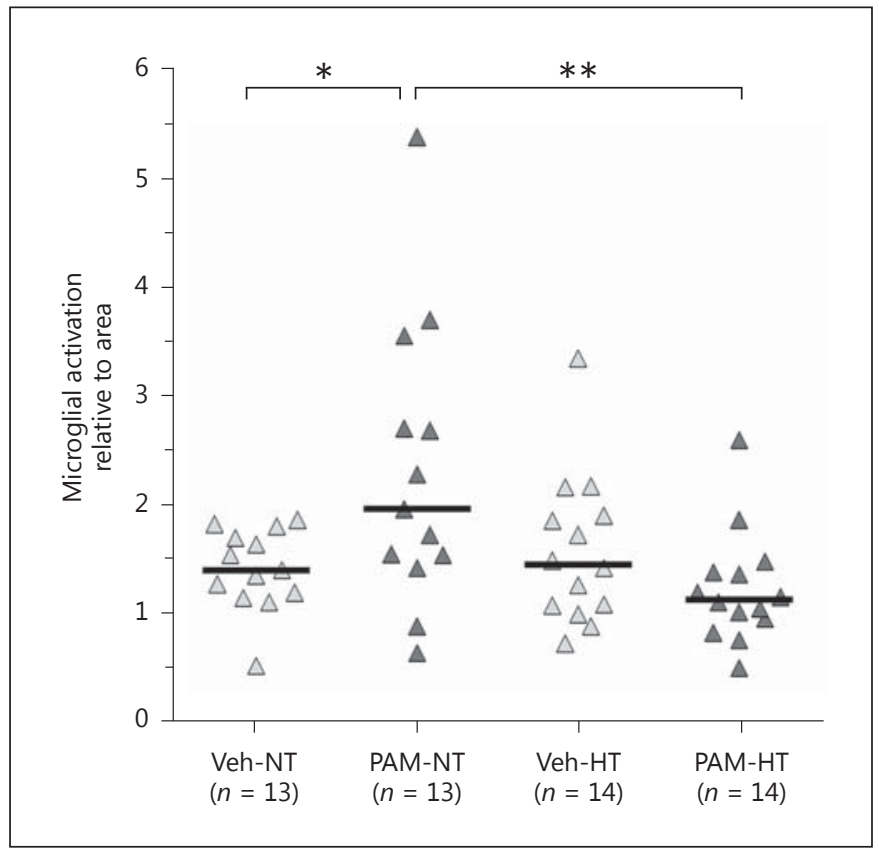

Fig. 5. Ibal density (microglial activation) relative to cross-sectional brain area. $\mathrm{PAM}_{3} \mathrm{CSK}_{4}$-injected (PAM) animals treated with NT (PAM-NT) have a greater degree of microglial activation relative to remaining tissue, compared to vehicle-injected (Veh) pups receiving the same treatment (Veh-NT). HT counteracts this effect. ${ }^{*} p=0.035,{ }^{* *} p=0.006$.

brain injury to more closely resemble the clinical situation in term asphyxiated neonates, where gram-positive infections predominate. Here we provide evidence that PAM, a TLR-2 agonist, does sensitise the immature brain when injected systemically. Importantly, PAM-injected animals are equally vulnerable to $\mathrm{HI}$ as those sensitised by LPS [8]. However, HT still provides $>80 \%$ neuroprotection of hemispheric area loss in animals administered PAM. This is in stark contrast to studies on LPS-induced sensitisation, where HT was ineffective.

Analyses of hippocampal area loss and neuron count in the CA1 region gave similar neuroprotection of HT after sensitisation with PAM. In the PAM groups, treatment with HT resulted in an $85 \%$ reduction of total hippocampal tissue loss, and a 2.4 -fold higher number of surviving neurons in the CA1 region of the hippocampus. Tissue loss in the cortex followed the same pattern across the groups, with again significant neuroprotection in PAM-HT animals. Interestingly, the thalamus is a less vulnerable area to the sensitizing effect of PAM, without worse outcome in the PAM-NT group compared to the Veh-NT group. Still hypothermia reduces injury signifi-

Pathogen-Dependent Hypothermic Neuroprotection
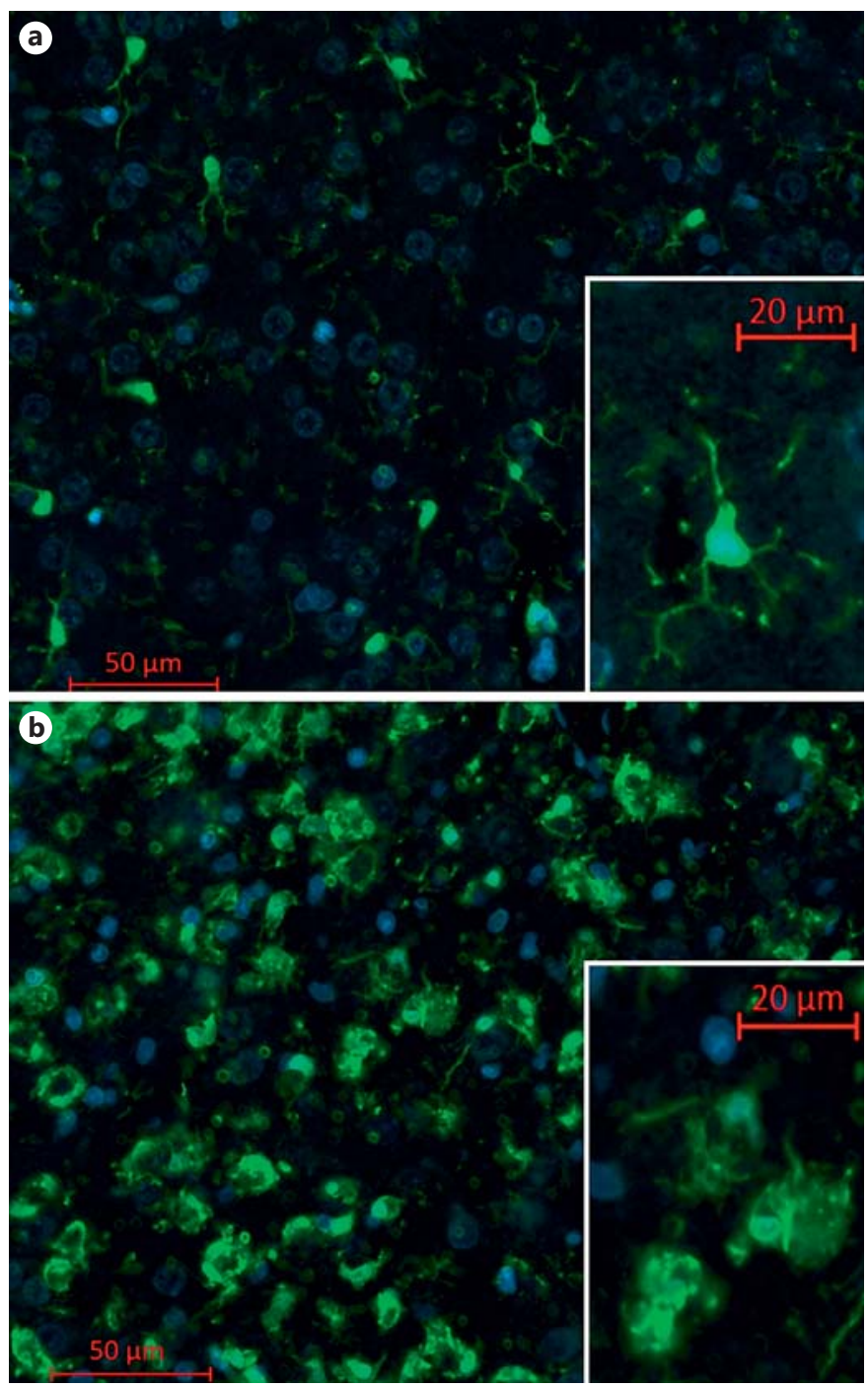

Fig. 6. Representative images of microglia stained for Iba1. a A Veh-NT animal showing ramified resting microglia with a small soma and long slender branched processes. b A PAM-NT animal demonstrating proliferation and upregulation of activated microglia, with big round soma and retracted processes.

cantly also in this region. Additionally, microglial activation relative to cross-sectional area was increased in PAM-sensitised brains. This was reversed by HT, with a $55 \%$ reduction of Ibal expression.

Injection of PAM or of LPS alone results in neuro-inflammatory alterations that differ highly depending on the inflammatory stimulus [35]. Our own experiments have shown that LPS-injected rat pups become hypothermic soon after injection, with a spontaneous core temperature drop of $3.5^{\circ} \mathrm{C}$, from $35^{\circ} \mathrm{C}$ down to $31.5^{\circ} \mathrm{C}$ [27].

Dev Neurosci 2017;39:238-247 243 
This decrease in core temperature was not seen in animals injected with PAM, which were not different from Veh animals. Intrahypoxic temperature is known to have a large impact on the susceptibility of the neonatal rat brain to HI injury [36]. At higher core temperatures during hypoxia, neonatal rats are more susceptible to brain injury, and vice versa, at lower body temperature it is more challenging to create a lesion [37]. The drop in core temperature we have seen after LPS injection, which is still present when the experiment commences, might partly be the reason for their increased vulnerability compared to Veh animals [5]. In studies on LPS sensitisation, the rats receive the insult at the same intrahypoxic temperature as control groups $\left(36^{\circ} \mathrm{C}\right)[5,8]$, meaning their temperature during hypoxia is rapidly increased by $4.5^{\circ} \mathrm{C}$ when placed in the hypoxia chamber. The temperature of Veh pups or juvenile control animals on the other hand is only increased by up to $1^{\circ} \mathrm{C}$ during the hypoxia period. This does, however, not explain why PAM-injected pups, which maintain the same core temperature as Veh animals after injection, present a vulnerability to hypoxia similar to that of LPS-injected pups. This suggests that other mechanisms are as important as temperature when it comes to the brain's resistance to an $\mathrm{HI}$ insult.

$\mathrm{HI}$ brain injury without systemic inflammation induces a lowering of core temperature $[36,38]$. During hypoxia there is also reduced metabolism and heat production [39]. The detailed mechanism behind this phenomenon, however, is not fully understood, and an innate neuroprotective defence mechanism has been suggested [36]. Experimental HI brain injury without infectious presensitisation is where HT has repeatedly been shown to be neuroprotective [40]. We therefore speculate whether the reduced core temperature found after injection of LPS might be more of a pathological response, with disturbance of the thermoregulatory centre in the hypothalamus. Linthorst et al. [41] have demonstrated several disturbances in the thalamic pre-optic area after intraperitoneal LPS administration, which substantiates this theory. These changes have not been investigated after PAM sensitisation. A study comparing these responses after PAM or LPS would help elucidate these mechanisms and their influence in infection-sensitised brain injury.

Resting microglia are activated in response to $\mathrm{HI}$ injury [42-44]. Studies on LPS-sensitised HI injury have demonstrated microglia to be both more numerous and in a more activated state around the site of the lesion [18, $45,46]$. The sensitising effect of LPS on the immature brain has been attributed to the number of activated microglia. Here we demonstrate a similar microglial re- sponse after PAM, with comparable increased neuronal vulnerability. This could suggest that microglial activation and proliferation is involved in the pathogenic inflammatory activation and brain sensitisation due to the combination of PAM and HI. It is however noteworthy that both area loss and microglial activation after PAMsensitised HI is largely reversed by HT, while this is not the case after LPS. Microglial activation seems to be nonspecific to the pathogen, and part of a more distal common pathway of neuro-inflammatory responses. In both models, the increased microglial response in sensitised brains is associated with a higher median degree of neuronal injury, due to the "double hit" insult, compared to controls. A constituent tonic inhibition of microglial activity occurs through ligand-receptor pairs from neurons, requiring direct cell-cell contact [44]. Even in the absence of damage-related signals, loss of neuronal integrity can induce a rapid microglial response [47]. The upregulation of Ibal could therefore represent a response to a more comprehensive injury, which occurred prior to the microglial activation. As these microglia are stained after 7 days' survival, and are not phenotyped, their activation state is likely to be towards what was previously defined as the M2 phenotype on the classification spectrum, and a sign of inflammatory repair mechanisms [48]. The dramatic difference in sensitivity to HT indicates that other, earlier, inflammatory events are more important in the mechanistic explanation of inflammatory presensitisation to neuronal Hi injury.

A limitation to this study is the lack of significant HT neuroprotection in the Veh-injected animals. We do however see a somewhat lower median hippocampal area loss in the Veh-HT group compared to the Veh-NT group, although it is not statistically significant. This goes well with how the hippocampus has been shown to be the area most sensitive to HI, but also the most sensitive to HT neuroprotection [49]. The lack of difference in hemispheric area loss may be due to the low degree of injury in this cohort as a result of the short hypoxia period (50 min compared to $90 \mathrm{~min}$ in our standard HI injury model without presensitisation) $[31,50]$. In our experience HT has not been neuroprotective after mild brain injury, defined in the Vannucci model as a median area loss below $25 \%[51,52]$. A moderate degree of injury $(30-60 \%$ tissue loss) is required to see a neuroprotective effect of HT in this model $[31,53]$. Whether mild injury should be eligible for cooling in neonates is still debated, as the neuroprotective effect of HT has not been clarified for these patients [54]. Rat pups are highly variable in how much hypoxia they can withstand before cellular death is seen, 
and the same is likely to be the case for human neonates. The well-described variability of injury in the Vannucci model demands a substantial sample size, and the careful use of non-parametric statistical approaches. However, this model has been an important part of translating therapeutic hypothermia from bench to bedside [55], and harbouring such variability might be part of what makes the model translatable. When we modify the model to include systemic infectious inflammatory activation, chances are high that processes and pathways are induced that still remain to be uncovered. The immune system is far from fully mapped, and furthermore, studies on translation of immune responses across species are scarce [56].

Though the focus of research on presensitisation has primarily been based around bacterial infections, general signs of infection are mostly non-specific and could be associated with other infectious agents. Some of the studies associating severity of brain injury to systemic inflammation used maternal fever as a sign of infection $[2,3]$, but the most common cause of fever is viral infections including influenza, rhinovirus, enterovirus and coronavirus [57]. The presensitising effect of viral-induced maternofetal inflammation has not been well investigated clinically. A study by Stridh et al. [58] on neonatal mice demonstrated a significantly increased infarct volume after presensitisation with an agonist to TLR-3, the pathogen recognition receptor of viral RNA. Fever is induced by raised circulating levels of certain cytokines, specifically IL-6 [59], which occurs during both bacterial and viral illnesses [60]. A well-known consequence of intrapartum maternal infection is the fetal inflammatory response syndrome, also characterised by elevated IL-6 levels in the fetus [61]. Whether this is dependent on the pathogen is not known and could indeed include maternal viral infections as well as bacterial chorio-amnionitis. With respect to how viral infections may interact with HT in asphyxiated neonates, it is interesting to note that infections with common cold viruses increase in the winter season, and cold viruses have been shown to replicate better at cold environmental temperatures like in the nasal cavity $\left(33-35^{\circ} \mathrm{C}\right)$ than at normal core temperature $\left(37^{\circ} \mathrm{C}\right)$. The mechanism behind this is not yet elucidated but is thought to be due to diminished antiviral immune responses at these lowered temperatures [62]. Cooling neonates with a viral infection might therefore bring them to a temperature that promotes the growth of certain viruses. The downstream effects of that are unknown.

Eklind et al. developed a modification of the Vannucci model with systemic inflammation to presensitise the brain to HI insult [5]. They used LPS as a systemic inflam-

Pathogen-Dependent Hypothermic

Neuroprotection matory trigger, and thereby modelled a gram-negative bacterial infection. This finding was particularly important with respect to the prematurely born population [63, 64] and furthermore to populations of less developed parts of the world, where the incidence of gram-negative infections is higher, and HT was shown not to have neuroprotective effect [65]. Our group found the same HIsensitising effect of LPS on the brain; however, we showed that HT neuroprotection was negated in LPS-sensitised rat pups using that model $[8,18]$. Studies on HT in lowincome settings have not been able to demonstrate a neuroprotective effect [21], and HT is to date standard of care only in western high-income countries. On this basis, and knowing our target patient group to mostly have infections caused by gram-positive bacteria, we further developed a model of gram-positive infection, using a synthetic TLR-2 antagonist, as described. Activation of TLR-2 triggers inflammatory activation through the same pathway that initiates sepsis from gram-positive species [26, 66]. Surprisingly, and in contrast to the results seen in the LPS model, we demonstrate a neuroprotective effect of HT. This might not uncover the whole story, but it does underline the importance of tailoring our preclinical models as thoroughly as we can to the clinical scenario we aim to mimic.

With these data we can only conclude that HT can be highly neuroprotective in inflammatory presensitised HI injury, but the neuroprotective effect might depend on the pathogen. With current knowledge, our results in combination with clinical infection demographics suggest that we should continue to treat encephalopathic neonates who fulfil the cooling criteria, regardless of infectious status.

\section{Acknowledgements}

This study was supported by the Norwegian Research Council (NFR 214356/F20). We are also grateful for additional support from the German Research Council (to H.S.). We thank Prof. Lars Walløe for advice on statistical analysis.

\section{Disclosure Statement}

The authors declare no competing financial interests.

Dev Neurosci 2017;39:238-247

DOI: $10.1159 / 000455838$ 


\section{References}

1 Jacobs SE, Berg M, Hunt R, Tarnow-Mordi WO, Inder TE, Davis PG: Cooling for newborns with hypoxic ischaemic encephalopathy. Cochrane Database Syst Rev 2013; 1:CD003311.

2 Eastman NJ, Deleon M: The etiology of cerebral palsy. Am J Obstet Gynecol 1955;69:950961.

3 Grether JK, Nelson KB: Maternal infection and cerebral palsy in infants of normal birth weight. JAMA 1997;278:207-211.

4 Strunk T, Inder T, Wang X, Burgner D, Mallard C, Levy O: Infection-induced inflammation and cerebral injury in preterm infants. Lancet Infect Dis 2014;14:751-762.

5 Eklind S, Mallard C, Leverin AL, Gilland E, Blomgren K, Mattsby-Baltzer I, et al: Bacterial endotoxin sensitizes the immature brain to hypoxic-ischaemic injury. Eur J Neurosci 2001;13:1101-1106.

6 Fleiss B, Tann CJ, Degos V, Sigaut S, Van Steenwinckel J, Schang A-L, et al: Inflammation-induced sensitization of the brain in term infants. Dev Med Child Neurol 2015; 57(suppl 3):17-28.

7 Hagberg H, Mallard C, Ferriero DM, Vannucci SJ, Levison SW, Vexler ZS, et al: The role of inflammation in perinatal brain injury. Nat Rev Neurol 2015;11:192-208.

8 Osredkar D, Thoresen M, Maes E, Flatebø T, Elstad M, Sabir H: Hypothermia is not neuroprotective after infection-sensitized neonatal hypoxic-ischemic brain injury. Resuscitation 2014;85:567-572.

9 Mallard C, Welin A-K, Peebles D, Hagberg H, Kjellmer I: White matter injury following systemic endotoxemia or asphyxia in the fetal sheep. Neurochem Res 2003;28:215-223.

10 Wang X, Stridh L, Li W, Dean J, Elmgren A, Gan L, et al: Lipopolysaccharide sensitizes neonatal hypoxic-ischemic brain injury in a MyD88-dependent manner. J Immunol 2009; 183:7471-7477.

11 Baburamani AA, Miyakuni Y, Vontell R, Supramaniam VG, Svedin P, Rutherford M, et al: Does caspase- 6 have a role in perinatal brain injury? Dev Neurosci 2015;37:321-337.

12 Chau V, Brant R, Poskitt KJ, Tam EW, Synnes A, Miller SP: Postnatal infection is associated with widespread abnormalities of brain development in premature newborns. Pediatr Res 2012;71:274-279.

13 Nelson KB, Dambrosia JM, Grether JK, Phillips TM: Neonatal cytokines and coagulation factors in children with cerebral palsy. Ann Neurol 1998;44:665-675.

14 Fjalstad JW, Stensvold HJ, Bergseng H, Simonsen GS, Salvesen B, Rønnestad AE, et al: Early-onset sepsis and antibiotic exposure in term infants: a nationwide population-based study in Norway. Pediatr Infect Dis J 2016;35: $1-6$.

15 Rousset CI, Kassem J, Aubert A, Planchenault D, Gressens P, Chalon S, et al: Maternal expo- sure to lipopolysaccharide leads to transient motor dysfunction in neonatal rats. Dev Neurosci 2013;35:172-181.

16 Hoogland ICM, Houbolt C, van Westerloo DJ, van Gool WA, van de Beek D: Systemic inflammation and microglial activation: systematic review of animal experiments. J Neuroinflamm 2015;12:114.

17 Ikeda T, Yang L, Ikenoue T, Mallard C, Hagberg $\mathrm{H}$ : Endotoxin-induced hypoxic-ischemic tolerance is mediated by up-regulation of corticosterone in neonatal rat. Pediatr Res 2006;59:56-60.

18 Osredkar D, Sabir H, Falck M, Wood T, Maes E, Flateb T, et al: Hypothermia does not reverse cellular responses caused by lipopolysaccharide in neonatal hypoxic-ischaemic brain injury. Dev Neurosci 2015;37:390-397.

19 Perlman JM, Wyllie J, Kattwinkel J, Atkins DL, Chameides L, Goldsmith JP, et al: Part 11: neonatal resuscitation: 2010 international consensus on cardiopulmonary resuscitation and emergency cardiovascular care science with treatment recommendations. Circulation 2010;122:S516-S538.

20 Edwards AD, Brocklehurst P, Gunn AJ, Halliday H, Juszczak E, Levene M, et al: Neurological outcomes at 18 months of age after moderate hypothermia for perinatal hypoxic ischaemic encephalopathy: synthesis and meta-analysis of trial data. BMJ 2010;340:c363.

21 Robertson NJ, Nakakeeto M, Hagmann C, Cowan FM, Acolet D, Iwata O, et al: Therapeutic hypothermia for birth asphyxia in lowresource settings: a pilot randomised controlled trial. Lancet 2008;372:801-803.

22 Wintermark P, Boyd T, Gregas MC, Labrecque M, Hansen A: Placental pathology in asphyxiated newborns meeting the criteria for therapeutic hypothermia. Am J Obstet Gynecol 2010;203:579. e1-e9.

23 Guha M, Mackman N: LPS induction of gene expression in human monocytes. Cell Signal 2001;13:85-94.

24 Yoshimura A, Lien E, Ingalls RR, Tuomanen E, Dziarski R, Golenbock D: Cutting edge: recognition of gram-positive bacterial cell wall components by the innate immune system occurs via Toll-like receptor 2. J Immunol 1999;163:1-5.

25 Takeuchi O, Hoshino K, Kawai T, Sanjo H, Takada H, Ogawa T, et al: Differential roles of TLR2 and TLR4 in recognition of gram-negative and gram-positive bacterial cell wall components. Immunity 1999;11:443-451.

26 Kumar H, Kawai T, Akira S: Pathogen recognition by the innate immune system. Int Rev Immunol 2011;30:16-34.

27 Falck M, Sabir H, Maes E, Wood TR, Flatebø $\mathrm{T}$, Osredkar D, et al: Moving towards a more clinically relevant animal model of infectionsensitized HIE. 2016. http://www.hersheyconference.com/ (accessed September 12, 2016).
28 Andrade EB, Alves J, Madureira P, Oliveira L, Ribeiro A, Cordeiro-da-Silva A, et al: TLR2induced IL-10 production impairs neutrophil recruitment to infected tissues during neonatal bacterial sepsis. J Immunol 2013;191: 4759-4768.

29 Stridh L, Ek CJ, Wang X, Nilsson H, Mallard C: Regulation of Toll-like receptors in the choroid plexus in the immature brain after systemic inflammatory stimuli. Transl Stroke Res 2013;4:220-227.

$30 \mathrm{Du}$ X, Fleiss B, Li H, D’Angelo B, Sun Y, Zhu C, et al: Systemic stimulation of TLR2 impairs neonatal mouse brain development. PLoS One 2011;6:e19583.

31 Sabir H, Scull-Brown E, Liu X, Thoresen M: Immediate hypothermia is not neuroprotective after severe hypoxia-ischemia and is deleterious when delayed by $12 \mathrm{~h}$ in neonatal rats. Stroke 2012;43:3364-3370.

32 Towfighi J, Mauger D, Vannucci RC, Vannucci SJ: Influence of age on the cerebral lesions in an immature rat model of cerebral hypoxia-ischemia: a light microscopic study. Brain Res Dev Brain Res 1997;100:149-160.

33 Gehrmann J, Bonnekoh P, Miyazawa T, Oschlies U, Dux E, Hossmann KA, et al: The microglial reaction in the rat hippocampus following global ischemia: immuno-electron microscopy. Acta Neuropathol 1992;84:588595.

34 Thoresen M, Bågenholm R, Løberg EM, Apriccna F: The stress of being restrained reduces brain damage after a hypoxic-ischaemic insult in the 7-day-old rat. Neuroreport 1996;7:481-484.

35 Mottahedin A, Smith PLP, Hagberg H, Ek CJ, Mallard C: TLR2-mediated leukocyte trafficking to the developing brain. J Leukoc Biol 2017;101:297-305.

36 Reinboth BS, Köster C, Abberger H, Prager S, Bendix I, Felderhoff-Müser U, et al: Endogenous hypothermic response to hypoxia reduces brain injury: implications for modeling hypoxic-ischemic encephalopathy and therapeutic hypothermia in neonatal mice. Exp Neurol 2016;283:264-275.

37 Yager J, Towfighi J, Vannucci RC: Influence of mild hypothermia on hypoxic-ischemic brain damage in the immature rat. Pediatr Res 1993;34:525-529.

38 Burnard ED, Cross KW: Rectal temperature in the newborn after birth asphyxia. BMJ 1958;2:1197-1199.

39 Dawkins MJR, Hull D: Brown adipose tissue and the response of new-born rabbits to cold. J Physiol 1964;172:216-238.

40 Thoresen M, Wyatt J: Keeping a cool head, post-hypoxic hypothermia - an old idea revisited. Acta Paediatr 1997;86:1029-1033.

41 Linthorst ACE, Flachskamm C, Holsboer F, Reul JMHM: Intraperitoneal administration of bacterial endotoxin enhances noradrenergic neurotransmission in the rat preoptic 
area: relationship with body temperature and hypothalamic-pituitary-adrenocortical axis activity. Eur J Neurosci 1995;7:2418-2430.

42 McRae A, Gilland E, Bona E, Hagberg H: Microglia activation after neonatal hypoxicischemia. Brain Res Dev Brain Res 1995;84: 245-252.

43 Li T, Pang S, Yu Y, Wu X, Guo J, Zhang S: Proliferation of parenchymal microglia is the main source of microgliosis after ischaemic stroke. Brain 2013;136:3578-3588.

44 Pierre WC, Smith PLP, Londono I, Chemtob S, Mallard C, Lodygensky GA: Neonatal microglia: the cornerstone of brain fate. Brain Behav Immun 2017;59:333-345.

45 Kim WG, Mohney RP, Wilson B, Jeohn GH, Liu B, Hong JS: Regional difference in susceptibility to lipopolysaccharide-induced neurotoxicity in the rat brain: role of microglia. J Neurosci 2000;20:6309-6316.

46 Dean JM, Shi Z, Fleiss B, Gunn KC, Groenendaal F, van Bel F, et al: A critical review of models of perinatal infection. Dev Neurosci 2015;37:289-304.

47 Hanisch U-K, Kettenmann H: Microglia: active sensor and versatile effector cells in the normal and pathologic brain. Nat Neurosci 2007;10:1387-1394.

48 Mantovani A, Sica A, Sozzani S, Allavena P, Vecchi A, Locati M: The chemokine system in diverse forms of macrophage activation and polarization. Trends Immunol 2004;25:677686.

49 Bona E, Hagberg H, Løberg EM, Bågenholm $\mathrm{R}$, Thoresen M, Loberg EM, et al: Protective effects of moderate hypothermia after neonatal hypoxia-ischemia: short- and long-term outcome. Pediatr Res 1998;43:738-745.

50 Hobbs C, Thoresen M, Tucker A, Aquilina K, Chakkarapani E, Dingley J: Xenon and hypothermia combine additively, offering long- term functional and histopathologic neuroprotection after neonatal hypoxia/ischemia. Stroke 2008;39:1307-1313.

51 Sameshima H, Ikenoue T: Hypoxic-ischemic neonatal encephalopathy: animal experiments for neuroprotective therapies. Stroke Res Treat 2013;2013:659374.

52 Ota A, Ikeda T, Ikenoue T, Toshimori K: Sequence of neuronal responses assessed by immunohistochemistry in the newborn rat brain after hypoxia-ischemia. Am J Obstet Gynecol 1997;177:519-526.

53 Wood T, Osredkar D, Puchades M, Maes E, Falck M, Flatebø T, et al: Treatment temperature and insult severity influence the neuroprotective effects of therapeutic hypothermia. Sci Rep 2016;6:23430.

54 Gagne-Loranger M, Sheppard M, Ali N, Saint-Martin C, Wintermark P: Newborns referred for therapeutic hypothermia: association between initial degree of encephalopathy and severity of brain injury (what about the newborns with mild encephalopathy on admission?). Am J Perinatol 2015;33:195-202.

55 Gunn AJ, Laptook AR, Robertson NJ, Barks JD, Thoresen M, Wassink G, et al: Therapeutic hypothermia translates from ancient history in to practice. Pediatr Res 2017;81:202209.

56 Seok J, Warren HS, Cuenca AG, Mindrinos MN, Baker H V, Xu W, et al: Genomic responses in mouse models poorly mimic human inflammatory diseases. Proc Natl Acad Sci USA 2013;110:3507-3512.

57 Fowlkes A, Giorgi A, Erdman D, Temte J, Goodin K, Di Lonardo S, et al: Viruses associated with acute respiratory infections and in- fluenza-like illness among outpatients from the Influenza Incidence Surveillance Project, 2010-2011. J Infect Dis 2014;209:1715-1725.

58 Stridh L, Mottahedin A, Johansson ME, Valdez RC, Northington F, Wang X, et al: Tolllike receptor-3 activation increases the vulnerability of the neonatal brain to hypoxiaischemia. J Neurosci 2013;33:12041-12051.

59 Leon LR, White AA, Kluger MJ: Role of IL-6 and TNF in thermoregulation and survival during sepsis in mice. Am J Physiol 1998; 275:R269-R277.

60 Akira S, Kishimoto T: IL-6 and NF-IL6 in acute-phase response and viral infection. Immunol Rev 1992;127:25-50.

61 Gotsch F, Romero R, Kusanovic JP, MazakiTovi S, Pineles BL, Erez O, et al: Ovid: the fetal inflammatory response syndrome. Clin $\mathrm{Ob}-$ stet Gynecol 2007;50:652-683.

62 Foxman EF, Storer JA, Fitzgerald ME, Wasik BR, Hou L, Zhao H, et al: Temperature-dependent innate defense against the common cold virus limits viral replication at warm temperature in mouse airway cells. Proc Natl Acad Sci USA 2015;112:827-832.

63 Weston EJ, Pondo T, Lewis MM, MartellCleary P, Morin C, Jewell B, et al: The burden of invasive early-onset neonatal sepsis in the United States, 2005-2008. Pediatr Infect Dis J 2011;30:937-941.

64 Hornik CP, Fort P, Clark RH, Watt K, Benjamin DK, Smith PB, et al: Early and late onset sepsis in very-low-birth-weight infants from a large group of neonatal intensive care units. Early Hum Dev 2012;88:S69-S74.

65 Hamer DH, Darmstadt GL, Carlin JB, Zaidi AK, Yeboah-Antwi K, Saha SK, et al: Etiology of bacteremia in young infants in six countries. Pediatr Infect Dis J 2015;34:e1-8.

66 Schwandner R, Dziarski R, Wesche H, Rothe M, Kirschning CJ: Peptidoglycan- and lipoteichoic acid-induced cell activation is mediated by Toll-like receptor 2. J Biol Chem 1999;274: 17406-17409.
Pathogen-Dependent Hypothermic

Neuroprotection
Dev Neurosci 2017;39:238-247

DOI: $10.1159 / 000455838$ 